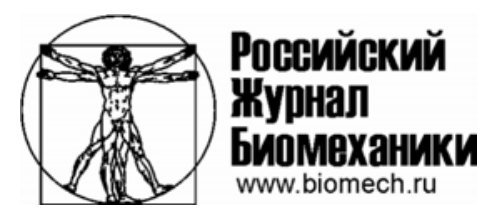

\title{
ОПТИМИЗАЦИЯ БИОМЕХАНИКИ СТИМУЛОВ ВАКУУМА В АППАРАТЕ С КОМПОНЕНТОЙ СЖАТИЯ ДЛЯ ВЫВЕДЕНИЯ МОЛОКА ИЗ МОЛОЧНОЙ ЖЕЛЕЗЫ ЛАКТИРУЮЩИХ ЖЕНЩИН
}

\author{
Н.П. Алексеев, В.И. Ильин \\ Кафедра общей фризиологии Санкт-Петербургского государственного университета, Россия, 199034, \\ Санкт-Петербург, Университетская наб., 7/9, e mail: ultra3@yandex.ru
}

\begin{abstract}
Аннотация. К настоящему времени установлено, что грудное молоко имеет значительные преимущества для развития и здоровья ребенка. При грудном вскармливании в ряде случаев женщина по разным причинам в течение некоторого времени не может кормить ребенка и, чтобы не прекратилась лактация, молоко должно сцеживаться. В настоящее время применяются два типа аппаратов. В первом типе молоко выводится только за счет импульсов вакуума, во втором - используются одновременно стимулы вакуума и сжатия. Работа этого аппарата в большей степени моделирует биомеханику выведения молока ребенком. Клинические испытания, а также многолетнее использование их в практике грудного вскармливания показали высокую стимулирующую способность этого типа аппаратов и более эффективное выведение молока по сравнению с вакуумными аппаратами. Однако временные характеристики стимулов, с помощью которых выводится молоко, и в частности вакуумных стимулов, остаются неоптимизированными. Была разработана методика, позволяющая сравнить количество молока, выведенного с помощью серии 0,5-секундных импульсов вакуума, с количеством молока, выведенного сериями вакуумных стимулов длительностью 0,3; 1 и 2 с, а также сравнивалось количество молока, выведенного серией импульсов 0,7 c, с количеством молока, выведенного серией импульсов вакуума длительностью 1 с. Молоко выводилось в течение одной сессии попеременно сериями импульсов различной длительности в различные емкости у одной и той же женщины. Для того чтобы влияние разницы в рефлекторном повышении давления в протоковой системе железы на выведение молока было минимально, периодически следующие друг за другом серии импульсов вакуума с различной длительностью стимулов имели продолжительность несколько секунд. Было обнаружено, что вакуумные стимулы длительностью 0,7 с и частотой 1 имп/с являются оптимальными для выведения молока из молочной железы женщины.
\end{abstract}

Ключевые слова: грудное вскармливание, рефрлекс выведения молока, молоковыводящие аппараты, биомеханика выведения молока, оптимизация стимулов вакуума.

\section{ВВеДЕНИЕ}

Имеющийся к настоящему времени большой экспериментальный материал свидетельствует, что грудное молоко важно для развития и здоровья ребенка, особенно для преждевременно родившихся детей [4-6]. Однако дети, родившиеся до 34-й недели беременности, еще не могут самостоятельно выводить молоко из молочной железы матери [6]. Поэтому женщины должны сцеживать молоко в зависимости от зрелости ребенка в течение нескольких недель или даже месяцев.

(С Алексеев Н.П., Ильин В.И., 2015

Алексеев Николай Петрович, д.б.н., профессор кафедры общей физиологии, Санкт-Петербург

Ильин Владимир Иванович, к.б.н., с.н.с. кафедры общей физиологии, Санкт-Петербург 
Кроме того, и у матерей, родивших детей в срок, могут возникать проблемы в процессе грудного вскармливания. Например, при болезни ребенка или матери, чтобы не прекратилась лактация, молоко должно сцеживаться. В большинстве случаев для сцеживания молока используются ручные или электрические молоковыводящие аппараты. В связи с этим, чтобы лактация женщины была успешной до того момента, когда ребенок может самостоятельно выводить молоко из груди, биомеханические характеристики аппарата должны удовлетворять ряду требований. Он должен: 1) эффективно механически стимулировать рецепторы ареолы молочной железы для формирования рефлексов выведения и секреции молока у лактирующей женщины; 2) не повреждать молочную железу и не вызывать болевых ощущений у женщины при выведении молока; 3) в достаточной степени быстро опорожнять железу от молока. В настоящее время применяются два типа аппаратов. В первом типе молоко выводится только за счет импульсов вакуума [7]. Во втором типе [1, 3] используются одновременно стимулы вакуума и сжатия. Работа этого аппарата в большей степени моделирует биомеханику выведения молока ребенком $[2,9]$. Клинические испытания, а также многолетнее использование их в практике грудного вскармливания показали высокую стимулирующую способность этого типа аппаратов и более эффективное выведение молока по сравнению с вакуумными аппаратами [1]. Вместе с тем необходимо отметить, что временные характеристики стимулов, с помощью которых выводится молоко, в частности вакуумных стимулов, остаются неоптимизированными.

Одними из главных критериев оценки эффективности стимулов исполнительного механизма аппарата являются количество молока и скорость выведения его из железы. Многочисленные исследования показали, что сцеживание молока у женщины в течение суток в значительной степени зависит от её физиологического и психологического состояния [7]. Поэтому оценить с достаточной точностью разницу в количестве молока, выведенного с помощью механического воздействия вакуумных стимулов различной длительности, можно, если молоко будет сцеживаться в одну и ту же сессию по очереди в разные емкости с использованием стимулов вакуума различной длительности. Однако здесь возникают трудности, связанные с импульсным характером рефлексов выведения молока. Под влиянием стимуляции механорецепторов ареолы молочной железы из центральной нервной системы женщины в кровяное русло периодически выделяется гормон окситоцин, который соответственно периодически увеличивает давление в протоковой системе молочной железы $[3,10]$. В предыдущих опытах авторов было обнаружено, что при сцеживании молока аппаратом внутрижелезистое давление периодически изменяется с длительностью пика повышения давления 1,5-2,0 мин. Изменяется также со временем амплитуда пиков и их последовательность. Причем у разных женщин эти параметры заметно отличаются [3, 10]. В связи с этим поочередное сцеживание вакуумными стимулами с различной длительностью в серии в разные емкости с длительностью серии импульсов, например 2 мин, будет вносить существенную неопределенность в результаты. Так, при сцеживании молока вакуумными стимулами одной длительности во время пика повышения давления в емкостной системе железы количество выведенного молока будет больше, чем при сцеживании вакуумными стимулами другой длительности в период наименьшего давления в емкостной системе. Поэтому, чтобы влияние разницы в давлении в протоковой системе железы на выведение молока было минимально, периодически следующие друг за другом серии импульсов вакуума с различной длительностью стимулов в серии должны иметь наименьшее время воздействия. Ранее авторами была разработана методика [1], которая позволяла для выведения молока воздействовать поочередно на сосок и ареолу короткими (4 с) сериями стимулов вакуума и сжатия. 
Целью данного исследования явилось модифицирование методики с тем, чтобы максимально уменьшить влияние эффекта рефлекторного повышения давления в протоковой системе молочной железы на разницу в количестве молока, выведенного импульсами вакуума различной длительности. С помощью данной методики провести обследования на молочных железах лактирующих женщин по оценке биомеханического влияния импульсов вакуума различной длительности на количество выведенного молока и скорость его выведения. Определить длительность вакуумных стимулов, при которой биомеханическое воздействие аппарата будет оптимально.

\section{МАТЕРИАЛЫ И МЕТОДЫ ИССЛЕДОВАНИЯ}

Обследование проводилось на 39 женщинах на 5-7-й день лактации, которые находились в послеродовом отделении Института акушерства и гинекологии им. Д.О. Отта РАН. Работа была выполнена с согласия этической комиссии Института акушерства и гинекологии им. Д.О. Отта РАН. Все обследованные женщины кормили детей грудью.

Оставшееся молоко после кормления сцеживалось аппаратом с компонентами вакуума и сжатия, конструкция которого была изменена в соответствии с целью работы. В частности, система, обеспечивающая выжимание молока из молочной железы, была отключена, и аппарат работал как обычный молокоотсос.

Аппарат состоит из блока управления с компрессором и выносной головки, которая накладывается на молочную железу женщины. На рис. 1 представлена схема выносной головки. На молочную железу 1 помещалась эластичная конусная накладка 2 , изготовленная из прозрачной силиконовой резины. Молоко из конусной накладки поступало в молокоотводящую силиконовую трубку 3, которая в свою очередь разделялась на две горизонтально располагающиеся трубки 4, сообщающиеся с отдельными молокосборниками. С одной стороны горизонтальные трубки прилегали к жесткой площадке 5, а с другой - контактировали с клинообразными подвижными штоками 6 пневмоуправляемых камер 7. При подведении в камеры давления штоки могли пережимать горизонтальные трубки и, соответственно, закрывать доступ молока в молокосборники. Ступенчатообразные вакуумные стимулы через электропневмоклапаны по трубкам 8 подавались в молокосборники и далее через горизонтальные трубки 4 и молокоотводящую трубку 3 внутрь эластичной конусной накладки. Для того чтобы исключить заброс молока из горизонтальных трубок 6

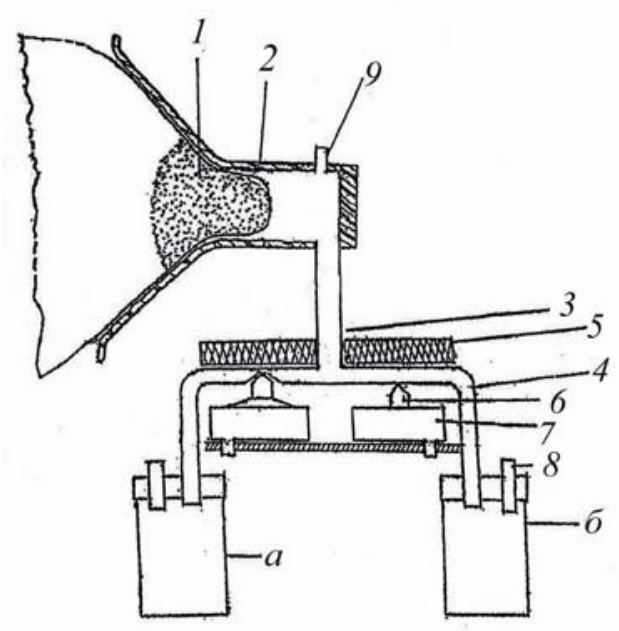

Рис. 1. Схема выносной головки аппарата: 1 сосок с ареолой молочной железы; 2 конусная накладка; 3 - молокоотводящая трубка; 4 - горизонтальная трубка; 5 - жесткая площадка; 6 - подвижной шток; 7 пневмоуправляемая камера; 8 - трубка, подводящая вакуум; 9 - патрубок для сброса вакуума внутри конусной накладки; $a, \sigma$ - молокосборники 
в молокоотводящую трубку 3 и внутрь конусной накладки, вакуум из конусной накладки сбрасывался через специальный патрубок 9, соединенный с помощью распределителя вакуума с атмосферой.

Система работает следующим образом. При включении аппарата в молокосборники поступали серии импульсов вакуума с определенной длительностью. В пневмоуправляемые камеры 7 поочередно подавались пневмоимпульсы давления, которые периодически на определенное время перекрывали ход молока и вакуума в горизонтальных трубках. Например, при перекрытии трубки, соединенной с молокосборником $a$, в молокосборник $\sigma$ под действием импульсов вакуума поступало молоко из железы. Затем трубка, соединенная с молокосборником $\sigma$, пережималась, и молоко поступало в молокосборник $a$.

Здесь следует отметить, что для получения точных данных определяющее значение имеет время действия последовательности вакуумных стимулов различной длительности. Обусловлено это, как уже отмечалось, особенностями рефлекторного выведения молока из железы лактирующей женщины. С целью минимизации влияния разницы рефлекторного внутрипротокового давления поочередное время действия вакуумных стимулов различной длительности было ограничено несколькими секундами. Кроме того, для двух сравниваемых серий импульсов вакуума различной длительности общее время действия серии импульсов вакуума на сосок и ареолу железы должно быть одинаковым. При этом в серию также должно укладываться целое число периодов обоих типов вакуумных импульсов (рис. 2). Длительность сессии выведения молока для каждой женщины была 20 мин.

Количество молока, выведенное с помощью вакуумных стимулов различной длительности, оценивалось в процентах к общему объему молока. Средние данные на гистограммах представлены со стандартным отклонением. Достоверность различия средних величин оценивали с помощью $t$-теста Стьюдента.

\section{РЕЗУЛЬТАТЫ ИССЛЕДОВАНИЙ И ИХ ОБСУЖДЕНИЕ}

В предварительных обследованиях была проверена симметричность функционирования молокопроводящих систем молокосборников $a$ и $б$. У 6 пациенток в качестве контроля молоко сцеживалось 0,5-секундными импульсами вакуума с промежутками между импульсами 0,5 с, амплитудой 120 мм рт. ст. и длительностью серии 4 с. Значение вакуума было выбрано на основании многочисленных обследований, которые показали отсутствие болевых ощущений у пациенток. Объем сцеженного молока в различных молокосборниках оценивали в процентах к общему количеству выведенного молока. На рис. 2, $A$ представлены гистограммы количества сцеженного молока в молокосборники $a$ и $\sigma$. Объем выведенного молока в молокосборнике $a$ был $49,5 \pm 2 \%$, а в молокосборнике $\sigma-50,5 \pm 1,5 \%(p \leq 0,05)$, т.е. был практически одинаков.

В первой части работы вакуумные стимулы длительностью 0,5 с, частотой 1 имп/с и амплитудой 120 мм рт. ст. были выбраны в качестве базовых. Количество молока, выведенное с помощью 0,5-секундных вакуумных стимулов, сравнивалось с объёмом молока, выведенного с помощью $0,3-; 1$ - и 2-секундных вакуумных стимулов (рис. $2,5-\Gamma$ ).

Для всех вакуумных стимулов в серии промежуток между импульсами, при котором давление внутри накладки было равно атмосферному (время отдыха), равнялся 0,5 с. Во второй части работы количество молока, выведенное с помощью 0,7-секундных вакуумных стимулов с промежутками между стимулами 0,3 с и длительностью серии 6 с, сравнивалось с объемом молока, выведенного с помощью 1,05-секундных вакуумных стимулов с промежутками между стимулами 0,45 с и длительностью серии 6 с (рис. 2, Д). 


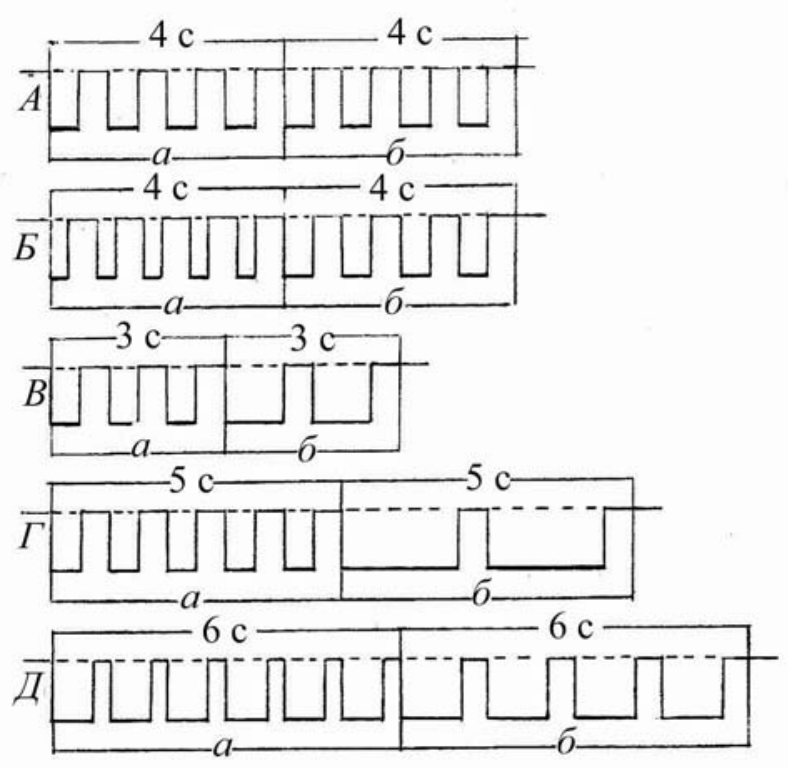

Рис. 2. Последовательность вакуумных стимулов, с помощью которых выводилось молоко. На $A-Д$ горизонтальными линиями сверху обозначено время серий импульсов, снизу обозначены серии импульсов, которые подавались в молокосборники $a$ и 6. Пунктирной линией показан атмосферный уровень давления. Отклонение импульсов вниз - вакуум

В первом обследовании в молокосборник $a$ поступало молоко, выведенное с помощью серий 0,5-секундных импульсов вакуума (4 импульса в серии). Трубка, ведущая в молокосборник 6 , в это время была перекрыта. Длительность серии -4 с, частота импульсов вакуума -1 имп/с. После того как пережималась горизонтальная трубка, соединенная с молокосборником $a$, открывалась трубка, ведущая в молокосборник $\sigma$ и в него поступало молоко, которое выводилось с помощью 0,3-секундных вакуумных стимулов (5 импульсов в серии). Длительность серии была также равна 4 с. Частота 0,3-секундных стимулов вакуума была 1,25 имп/с. После окончания серии 0,3-секундных вакуумных стимулов трубка, ведущая в молокосборник $\sigma$, пережималась, а в молокосборник $a$ открывалась, и цикл повторялся. Общая длительность вакуума в серии с 0,5-секундными импульсами превышала на 0,5 с общую длительность серии 0,3-секундных (рис. 2, Б). Поскольку молоко из железы выводилось только при действии стимулов вакуума, соотношение между длительностью вакуума $t_{v}$ к общей длительности серии $t_{s}$ могло быть показателем эффективности выбранного режима работы аппарата: $E=t_{v} / t_{s}$. В случае 0,3-секундных импульсов вакуума отношение суммы действия 0,3-секундных стимулов вакуума 1,5 с к общей длительности серии 4 с было $0,37 \quad(E=0,37)$. Отношение суммы действия 0,5-секундных стимулов вакуума 2 с к общей длительности серии 4 с было $0,5(E=0,5)$. Количество молока, выведенного за всю сессию сцеживания, было на $13 \%$ больше для 0,5-секундных стимулов вакуума, чем для 0,3 -секундных $(p \geq 0,05)$ (рис. $2, Б$ ). Поскольку время выведения молока в обоих случаях было одинаково, то и скорость выведения молока в случае 0,5-секундных импульсов была больше.

При аналогичном методе сравнения действия 3-секундной серии 0,5-секундных стимулов вакуума с 3-секундной серией 1-секундных импульсов вакуума (рис. 2, B) количество молока, выведенного с помощью 1-секундных стимулов вакуума, было 
примерно на $20 \%$ больше, чем с 0,5-секундными импульсами вакуума $(p \geq 0,05)$ (рис. $2, B$ ). При этом разница общего времени действия 1-секундных импульсов вакуума также превышала на 0,5 с общее время действия 0,5-секундных стимулов вакуума (рис. $2, B$ ). Для 0,5-секундных стимулов $E=0,5$, а для 1 -секундных $-E=0,67$. Частота 0,5-секундных импульсов вакуума в течение сессии равнялась 1 имп/с, а 1-секундных соответственно 0,67 имп/с.

Сравнение действия 5-секундной серии 0,5-секундных стимулов вакуума с 5-секундной серией 2-секундных импульсов вакуума (рис. 2, Г) на количество сцеженного молока обнаружило, что объем молока, выведенного с помощью 2 -секундных стимулов вакуума, был на $25,8 \%$ больше, чем 0,5 -секундных $(p \geq 0,05)$ (рис. $2, \Gamma$ ). Разница общего времени действия 2-секундных импульсов превышала на 1,5 с общее время действия 0,5-секундных (рис. 2, Г). Для 0,5-секундных вакуумных стимулов $E=0,5$, а для 2-секундных $-E=0,8$. Частота 0,5-секундных импульсов вакуума в течение сессии равнялась 1 имп/с, а 2-секундных соответственно 0,4 имп/с.

Таким образом, проведенные обследования показали, что при увеличении значений параметра $E$ количество и скорость выведения молока увеличиваются. Обусловлено это повышением соотношения между длительностью действия импульсов вакуума и промежутком (временем отдыха) между импульсами вакуума. Наибольшее значение $E$ может быть равно 1 . То есть в том случае, когда время отдыха становится равным 0, на сосок и ареолу действует непрерывный вакуум. Здесь необходимо указать, что в первых моделях молоковыводящих аппаратов молокоотсосах - использовался постоянный вакуум. Однако это вызывало болевые ощущения у женщин и сильный отек соска и ареолы [7]. Следует отметить, что в данной работе при сцеживании молока в сессии с 2-секундными импульсами вакуума у некоторых женщин также отмечались болевые ощущения и увеличивалась отечность ареолярно-сосковой области железы. Несмотря на отсутствие разницы во времени действия 0,3-0,5 с и 0,5-1,0 с импульсов вакуума, в серии отмечалась большая разница в объеме и скорости выведенного молока для второго случая (рис. 3, Б, B).

При разнице во времени действия в 1,5 с между сериями 0,5- и 2- секундных стимулов вакуума, не отмечалось пропорционального увеличения количества молока и скорости для серий 2-секундных вакуумных стимулов (рис. 3, Г). Эти данные позволяют говорить, что протоковая система молочной железы по своим биомеханическим свойствам «настроена» на выведение молока вакуумными стимулами определенной длительности. Кроме того, дополнительно можно предположить, что снижение эффективности выведения молока при увеличении длительности вакуумных стимулов до 2 с связано с возникновением у женщин неприятных ощущений и боли сосково-ареолярной области. Это может негативно влиять на центральные механизмы формирования рефлекса выведения молока и, соответственно, снизить эффективность сцеживания [11]. Здесь данные авторов согласуются с результатами исследований по влиянию формы вакуумных стимулов молокоотсосов на процесс выведения молока [8]. Оценивая болевые ощущения при действии стимулов вакуума в случае сцеживания молока импульсами вакуума различной формы и длительности, отметим, что менее комфортно себя чувствовали женщины при 2-3-секундных импульсах вакуума, используемых в экспериментальном молокоотсосе. Можно предположить, что увеличение межимпульсного интервала (время отдыха), например, до 1 с уменьшит степень дискомфорта длительных вакуумных импульсов. Однако в нашем случае для 2 -секундных импульсов $E$ снизится с 0,8 при 0,5-секундном интервале до 0,67 , т.е. будет такой же, как при 1-секундных импульсах вакуума. 


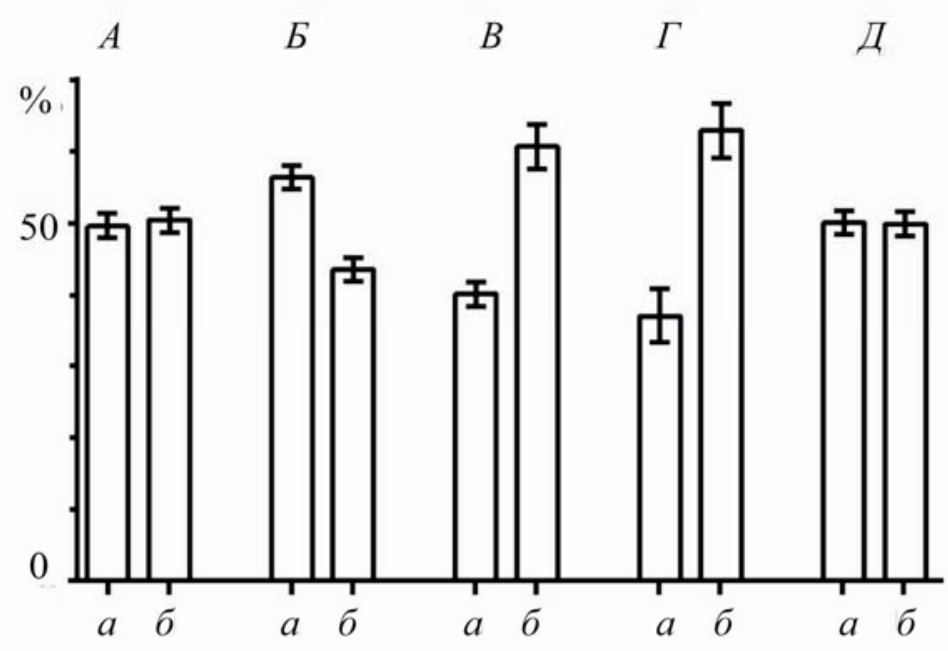

Рис. 3. Объём молока в молокосборниках $a$ и б, выведенного стимулами вакуума различной длительности, в процентах к общему количеству молока. Количество молока в молокосборниках, выведенного вакуумными стимулами длительностью $A-0,5$ с $(a)$ и 0,5 с (б); $Б-0,5$ с (a) и 0,3 с (б); $B-0,5$ с (a) и 1 с (б); $\Gamma-0,5$ с (a) и 2 с (б); Д- 0,7 с (a) и 1,05 с (б). По оси ординат объём молока в молокосборниках в процентах к общему количеству выведенного молока

Уменьшать длительность импульсов вакуума с соответствующим повышением их частоты с тем, чтобы увеличить общее время вакуума, также малоэффективно. Так, например, в случае уменьшения межимпульсного интервала в серии 0,3-секундных импульсов до 0,3 с $E$ повысится до 0,5 . При дальнейшем уменьшении межимпульсного интервала вакуумные импульсы практически сольются в непрерывный вакуум, что, несомненно, вызовет у женщин сильное чувство дискомфорта и боли. Вышеизложенное позволяет утверждать, что оптимальная для выведения молока длительность вакуума находится в области 1 с. Для проверки были выбраны вакуумные стимулы 0,7 с с интервалом между импульсами 0,3 с так, что частота в серии была 1 имп/с. Эффективность действия 0,7-секундных стимулов сравнивалась с 1,05-секундными стимулами вакуума с промежутком между стимулами 0,45 с и частотой 0,67 имп/с (см. рис. 2, Д). Количество 0,7-секундных стимулов вакуума в серии было 6, а число 1,05-секундных импульсов вакуума в серии - 4. Общее время действия 0,7- секундных импульсов вакуума и общее время действия 1,05- секундных стимулов вакуума было одинаково - 4,2 с (рис. 2, Д). Общая длительность серий импульсов в обоих случаях была 6 с. Для 0,7-секундных вакуумных стимулов $E=0,7$, a для 1,05-секундных $E=0,66$. Количество молока, выведенного за всю сессию сцеживания, было для 0,7-секундных стимулов вакуума $50,1 \pm 1,8 \%$ от общего количества, а для 1,05-секундных - 49,9 $\pm 1,8 \%$ (см. рис. 3, Д). Статистически достоверная разница отсутствовала $(p \leq 0,05)$. Вместе с тем женщины чувствовали меньший комфорт при сцеживании 1,05-секундными импульсами вакуума. Кроме того, отечность ареолы и соска была более выражена также при выведения молока 1,05-секундными стимулами вакуума.

\section{Выводы}

Представленные выше данные указывают на то, что оптимальная длительность импульсов вакуума для выведения молока из железы лактирующей женщины равняется 0,7 с при промежутке между импульсами 0,3 с и, соответственно, частоте 1 имп/с. 
Использование вакуумных импульсов такой длительности в аппаратах с выжимающей компонентой [1] обеспечит более эффективное выведение молока из железы лактирующих женщин.

\section{СПИСОК ЛИТЕРАТУРЫ}

1. Алексеев Н.П., Ильин В.И., Талалаева Н.Е. Вклад выжимающей компоненты исполнительного механизма молоковыводящего аппарата в процесс выведения молока у лактирующих женщин // Журнал акушерства и женских болезней. - 2014. - № 4. - С. 91-97.

2. Алексеев Н.П., Омельянюк У.В., Талалаева Н.Е. Изменение временных параметров стимулов сжатия при выведении молока во время кормления ребенка // Российский физиологический журнал им. И.М. Сеченова. - 2003. - № 11. - С. 1396-1403.

3. Алексеев Н.П., Ярославский В.К., Гайдуков С.Н., Ильин В.И., Спесивцев Ю.А., Тихонова Т.К., Кулагина Н.Б. Роль вакуумных и тактильных стимулов в процессе выведения молока из молочной железы женщины // Российский физиологический журнал им. И.М. Сеченова. - 1994. - № 10. С. 67-74.

4. Рюмина И.И., Ильин В.И. Профилактика и лечение гипогалактии: пособие для врачей / Моск. науч.исслед. ин-т педиатрии и дет. хирургии Министерства здравоохранения РФ. - М., 2000. - 11 с.

5. American academy of pediatrics. Breastfeeding and the use of human milk // Pediatrics. - 2012. Vol. 129, № 3. - P. e827-e841.

6. Fewtrell M.S., Lucas P., Collier S., Singhal A., Ahluwalia J.S., Lucas A. Randomized trial comparing the efficacy of a novel manual breast pump with a standard electric breast pump in mothers who delivered preterm infants // Pediatrics. - 2001. - Vol. 107, № 6. - P. 1291-1297.

7. Lawrence R.A., Lawrence R.M. Breastfeeding: a guide for medical profession. - 7th ed. - Philadelphia: Elsevier/Mosby, 2005. - 1114 p.

8. Mitoulas L.R., Lai C.T., Gurrin L.C., Larsson M., Hartmann P.E. Effect of vacuum profile on breast milk expression using an electric breast pum // J. Hum. Lact. - 2002. - Vol. 18. - P. 353-360.

9. Mizuno K., Ueda A. Development of sucking behavior in infants with Down's syndrome // Acta Paediatr. - 2000. - Vol. 90, № 12. - P. 1384-1388.

10. Prime D.K., Geddes D.T., Spatz D.L., Robert M., Trengove N.J., Hartmann P.E. Using milk flow rate to investigate milk ejection in the left and right breasts during simultaneous breast expression in women // Int. Breastfeed. J. - 2009. - Vol. 4, № 10. - P. 1-10.

11. Ueda T., Yokoyama Y., Irahara M., Aono T. Influence of psychological stress on suckling-induced pulsatile oxytocin release // Obstet. Gynecol. - 1994. - Vol. 84. - P. 259-262.

\section{OPTIMIZATION OF BIOMECHANICS OF THE VACUUM STIMULI IN BREAST PUMP WITH COMPRESSION COMPONENT}

\section{N.P. Alekseev, V.I. Ilyn (Saint-Petersburg, Russia)}

Breastfeeding due to its nutritional and immunological aspects is the best source of food for the newborn. In situation where breastfeeding is not possible, it is necessary to express milk from the breasts and better with by breast pump. Currently in medical practice, there are two types of breast pumps. In the first type, the milk is expressed only by vacuum impulses. In the second type, at the same time the stimuli of vacuum and compression are used in pumps. The operation of this apparatus largely simulates biomechanics of ejection of milk by children and has shown their higher efficacy compared with vacuum pumps. However, it should be noted that temporary characteristics of the stimuli, which are used for milk ejection and in particular the vacuum impulses are not optimized. The aim of this study was to determine the duration of vacuum stimuli in which biomechanical effects of the breast pump will be optimal. The amount of milk expressed by series of $0.5 \mathrm{~s}$ of vacuum stimuli were compared with the amount of milk extracted using series of $0.3,1,2 \mathrm{~s}$ vacuum stimuli 
and the amount of milk expressed by series of $0.7 \mathrm{~s}$ of vacuum stimuli were compared with the amount of milk extracted using series of $1 \mathrm{~s}$ vacuum stimuli. To minimize the influence of difference of reflex intramammary pressure, the times of the alternation series of vacuum stimuli of different duration was limited to a few seconds. It was found that vacuum stimuli duration of $0.7 \mathrm{~s}$ and a frequency of $1 \mathrm{imp} / \mathrm{s}$ are optimal for removing milk from the breast of a woman.

Key words: breastfeeding, milk ejection reflex, breast pump, biomechanics of milk ejection, optimization of vacuum stimuli.

Получено 12 июня 2015 